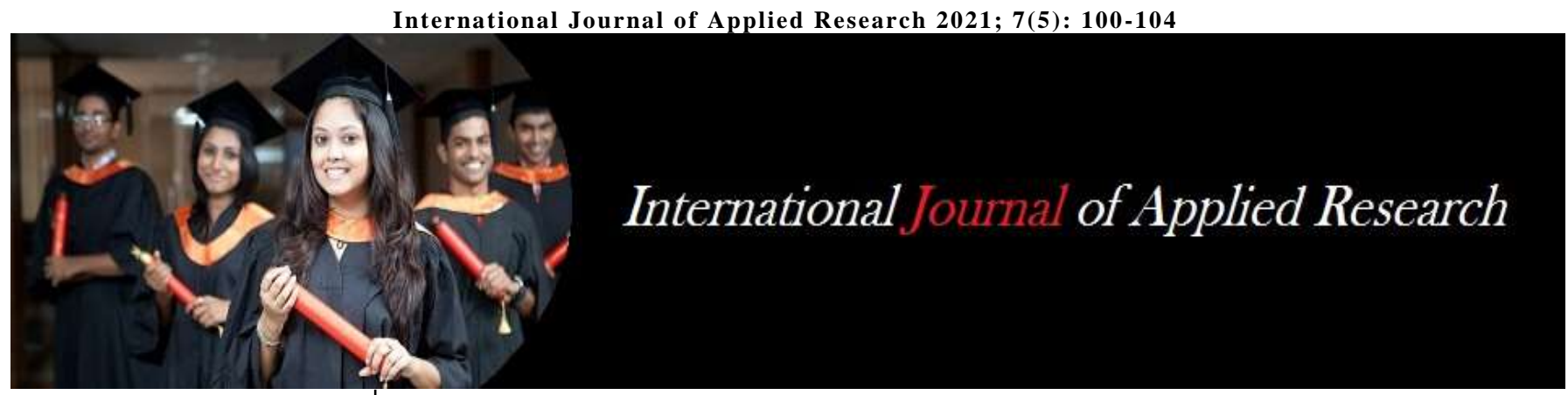

ISSN Print: 2394-7500

ISSN Online: 2394-5869

Impact Factor: 8.4

IJAR 2021; 7(5): 100-104

www.allresearchjournal.com

Received: 19-03-2021

Accepted: 21-04-2021

Sadguru Prakash

Department of Zoology,

MLKPG College, Balrampur,

Uttar Pradesh, India

Alok Shukla

Department of Physics,

MLKPG College, Balrampur,

Uttar Pradesh, India

\section{G and its impact on environment, biodiversity and human health: An overview}

\author{
Sadguru Prakash and Alok Shukla
}

DOI: https://doi.org/10.22271/allresearch.2021.v7.i5b.8552

\section{Abstract}

5G stands for the fifth generation of wireless technology. Millimetre waves are a higher frequency wavelength than the radio wavelength generally used today for transmission of information through this wireless technology. Both the wireless infrastructure of today and the modern era of 5G are seen as an asset in the intelligent system. It has been widely implemented throughout the world without discuss its adverse effects on future generations. It is argued that the addition of this added high-frequency 5G radiation to an already complex mix of lower frequencies, will contribute to a negative public health outcome both from both physical and mental health perspectives. This paper presents the adverse effects of $5 \mathrm{G}$ radiations on human health, plant and ecosystem. The thermal factors are also discussed in this article.

Keywords: Radio frequency, wireless communication, energy efficiency, specific absorption rate, millimetre wave, $5 \mathrm{~g}$ issues, environmental issues

\section{Introduction}

Fifth generation $(5 \mathrm{G})$ of communication technology promises to give faster speeds and a higher capacity for the use of more devices. It has been widely implemented throughout the world without discuss its adverse effects on environment, biodiversity and human health. In a time when the environment is at its most delicate, overlooking these impacts are extremely risky for future generations.

The main environmental issues associated with the implementation of the $5 \mathrm{G}$ network come with the manufacturing of the many component parts of the $5 \mathrm{G}$ infrastructure. In addition, the proliferation of new devices that will use the $5 \mathrm{G}$ network that is tied to the acceleration of demand from consumers for new 5G-dependent devices will have serious environmental consequences.

In fact, several scientists had started protesting against $5 \mathrm{G}$ at a very early stage of the technology. In 2015, 215 scientists from 41 countries communicated their alarm to the United Nations (UN) and World Health Organization (WHO). They stated that electromagnetic field adversely affects the plants animals, including ants, Birds, Forests, Frogs, Fruit flies, Honey bees, Insects, Mammals, Mice, Rats and human health. Effects include: Alteration of heart rhythm, altered gene expression, altered metabolism, altered stem cell development, cancers, cardiovascular disease, cognitive impairment, DNA damage, impacts on general well-being, increased free radicals, Learning and memory deficits, Impaired sperm function and quality, Miscarriage, Neurological damage, Obesity and diabetes and Oxidative stress. Effects in children include autism, attention deficit hyperactivity disorder (ADHD) and asthma.

\section{What is 5G?}

All wireless devices use electromagnetic spectrum radio frequencies in order to transmit information. $5 \mathrm{G}$ is known as the Internet of Things (IoT) platform infrastructure, where machine to machine communication possible. $5 \mathrm{G}$ stands for the fifth generation of wireless technology. WIFI routers, for example, occupy a range of bands from $900 \mathrm{MHz}$ to $60 \mathrm{GHz}$. The higher the frequency, the faster the information can be transmitted. It is the wave of wireless technology surpassing the $4 \mathrm{G}$ network that is used now.
Corresponding Author: Sadguru Prakash Department of Zoology, MLKPG College, Balrampur, Uttar Pradesh, India 
$4 \mathrm{G}$ and LTE use the sub-6 GHz frequency range, spanning from $700 \mathrm{MHz}$ to $2.7 \mathrm{GHz}$. $5 \mathrm{G}$ promises greater speeds and more bandwidth for users than $4 \mathrm{G}$ or LTE can offer. Previous generations brought the first cell phones
$(1 \mathrm{G})$, text messaging $(2 \mathrm{G})$, online capabilities $(3 \mathrm{G})$, and faster speed $(4 \mathrm{G})^{[1]}$. The waves in use now $4 \mathrm{G}$ can measure up to tens of centimetres, while the new $5 \mathrm{G}$ waves would be no greater than ten millimetres.

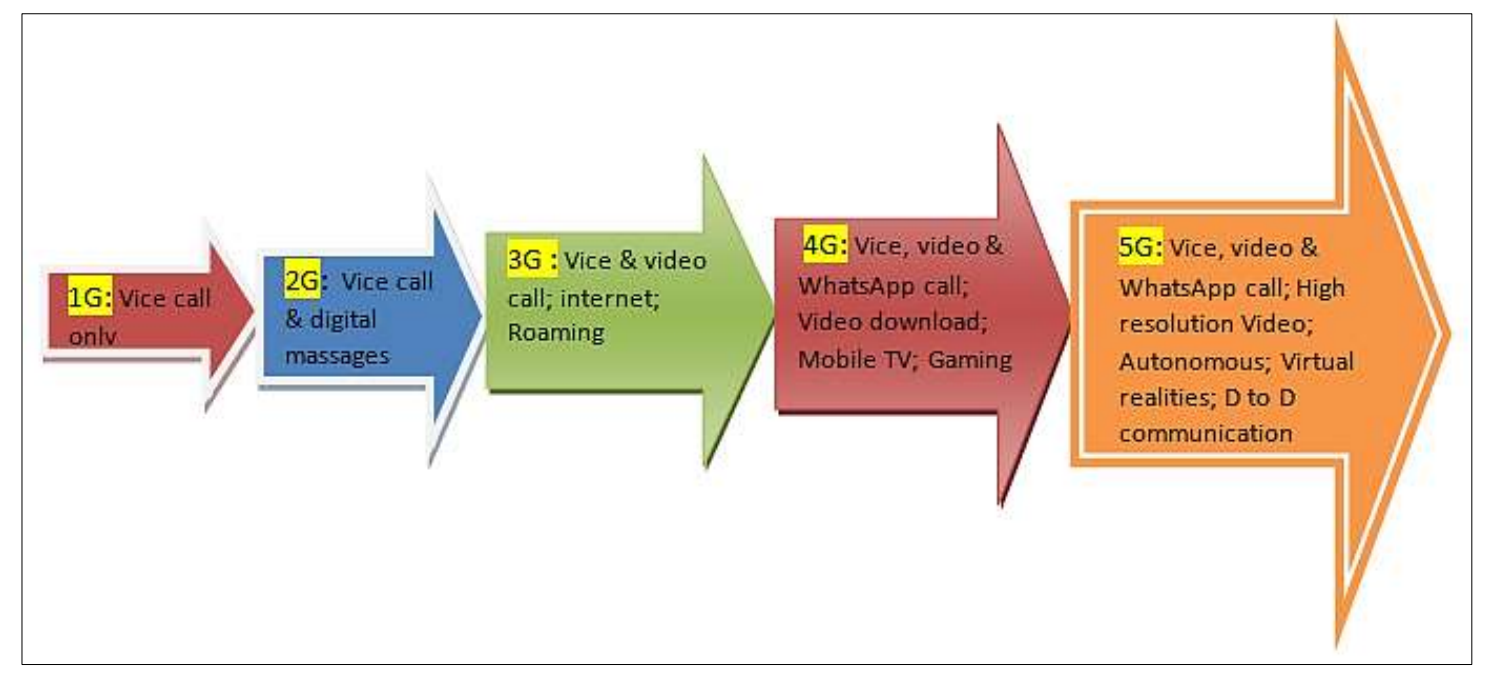

Fig 1: Generation of wireless network

The electromagnetic spectrum is the range of all types of electromagnetic radiation. The radio spectrum is the part of the spectrum used for telecommunication, broadcast, aircraft communication and more, and ranges from 30 hertz $(\mathrm{Hz})$ to 300 gigahertz ( $1 \mathrm{GHz}$ is equal to 1 billion hertz). The overall spectrum also includes visible light, gamma rays, x-rays, microwaves, etc. Spectrum on the lower end, called lowband (600 million hertz $(\mathrm{MHz})$ to $900 \mathrm{MHz}$ ) has longer waves and can travel farther. As waves range from midband $(2.5 \mathrm{GHz}$ to $4.2 \mathrm{GHz})$ to high-band, also known as millimetre wave $(24 \mathrm{GHz}$ to $47 \mathrm{GHz})$.

While low-band can penetrate walls well, its speed is limited to 100 megabytes per second (Mbps). Mid-band spectrum speed can reach 1 billion bytes or 1 gigabyte per second (Gbps); it has lower latency than low-band, but it cannot go through buildings as easily. High-band or millimetre wave
(mmWave) has very low latency and is super-fast, up to 10 Gbps. These high frequency mmWaves also offer increased transmission space so more devices can be connected at once. The drawback is that they are weaker and cannot easily penetrate solids. $5 \mathrm{G}$ operates on all three spectrum bands.

These millimetre waves are a higher frequency wavelength than the radio wavelength generally used in $5 \mathrm{G}$ wireless transmission technology today. The new technologies 5G will use are hardware that works with much higher frequencies (millimetre wavelengths/mmWave), small cells, massive MIMO (multiple input multiple output), beam forming, and full duplex ${ }^{[2]}$. Under different frequency bands, the $5 \mathrm{G}$ networks would operate with the lower frequencies shown in Table 1.

Table 1: Sub-division of $5 \mathrm{~g}$ frequency and its uses (Zaki et al., 2020) ${ }^{[3]}$

\begin{tabular}{|c|c|c|}
\hline Frequency range & Uses & Comments \\
\hline$<1 \mathrm{GH}$ & IoT & Longer range coverage, less costly infrastructure \\
\hline $1-6 \mathrm{GHz}$ & IoT, Capacity for transfer data & $\begin{array}{c}\text { More bandwidth, shorter range, and decreased efficiency compared with } \\
\text { higher frequencies }\end{array}$ \\
\hline$>6 \mathrm{GHz}$ & Extremely high Capacity for data transfer & Short-range, extremely high-speed data transfer and short latency \\
\hline
\end{tabular}

Many of these frequencies have previously been used by earlier forms of cell communications under $1 \mathrm{GHz}$ with Ultra-high frequencies (UHF). In comparison, even higher radio frequencies (RF) can still be seen in the later stages of technical improvements. The new bands have a higher frequency than UHF, with ranges of 3 to $26 \mathrm{GHz}$ in centimetre/millimetre ranges. The latter bands were usually used for radars and microwave connections ${ }^{[3]}$. The 5G deployment proposes to add frequencies in the microwave spectrum in the low- $(0.6 \mathrm{GHz}-3.7 \mathrm{GHz})$, mid- $(3.7 \mathrm{GHz}-$ $24 \mathrm{GHz}$ ), and high-band frequencies (24 GHz and higher) for faster communications ${ }^{[4]}$.

The millimetre wave spectrums of frequencies $24.25 \mathrm{GHz}$ and above (higher frequency wavelength than the radio wavelength) are generally used in wireless transmission today. Higher frequency waves allow for more devices to be connected to the same network at the same time, because there is more space available compared to the radio waves that are used today.

The aims of $5 \mathrm{G}$ network are to allow for more devices to be used by the consumer at faster rates than ever before ${ }^{[5]}$. This means that $5 \mathrm{G}$ will allow for nearly instantaneous downloading of data that, with the current network, would take hours. For example, downloading a movie using $5 \mathrm{G}$ would take mere seconds. The millimetre waves of $5 \mathrm{G}$ will create more space for devices to be used by consumers, which will certainly be an increase in energy usage globally, subsequently leading to increased global warming. Energy usage is one of the main contributors to climate change today and an increase in energy usage would cause climate change to increase drastically as well. 


\section{Effect of $5 \mathrm{G}$ radiation on human health}

The enormous demand increase for cell data would permit an unparalleled transmission power usage of the 5th generation $(5 \mathrm{G})$ broadband network in the millimetre wave (mm-Wave) bands. Millimetre waves are mostly absorbed within 1 to 2 millimetres of corneal surface layers and human skin. So, the cells or tissues of epidermis/ skin are the primary targets of the radiation. As the skin contains capillaries and nerve endings, millimetre waves bio-effects can be transmitted through molecular pathway by the skin or through the nervous system ${ }^{[6]}$. Exposure to the $5 \mathrm{G}$ radiation increases the production of reactive oxygen species (ROS are a normal part of cellular processes and cell signalling). Overproduction of ROS is not balanced either by endogenous antioxidants (superoxide dismutase, catalase, glutathione peroxidise, glutathione, melatonin), or exogenous antioxidants (Vitamin C, Vitamin E, carotenoids, polyphenols), so allows the formation of free radicals that oxidize and damage the DNA, proteins, membrane lipids and mitochondria. Mitochondrial doesn't have histones has a result of which it can't repair DNA damage and is not protected from mitochondrial reactive oxygen species ${ }^{[7]}$. Oxidative damage from ROS has been increasingly linked to the development and/or exacerbation of a number of chronic diseases and cancer. Excess ROS is produced due to radiation exposure, have been associated with exposure to toxic chemicals, pesticides and metals ${ }^{[4]}$. 5G spectrum might also induce electromagnetic sensitivity that has the following characteristics headaches, insomnia, dizziness, nausea, lack of concentration, heart palpitations, and depression.

Table 2: Electromagnetic Field (EMF) effect on health (Zaki et al., 2020) ${ }^{[3]}$

\begin{tabular}{|c|c|c|}
\hline Short-term effect & Long-term effect & Electrical sensitivity \\
\hline Aches and Pains & Cancer & Sleeping problems \\
\hline Headaches & Brain tumours & Cognitive impairment \\
\hline $\begin{array}{c}\text { Decreases sperm } \\
\text { motility }\end{array}$ & Fragmented DNA & $\begin{array}{c}\text { Concentration or } \\
\text { memory loss }\end{array}$ \\
\hline $\begin{array}{c}\text { Tingling or burning } \\
\text { sensations }\end{array}$ & Mutated cells & Brain Fog \\
\hline $\begin{array}{c}\text { Anxiety, stress, } \\
\text { irritability }\end{array}$ & Neurological & Anxiety and Mood \\
\hline
\end{tabular}

\section{Effect on skin}

The high spectrum range of $5 \mathrm{G}$ can leads mutation of cells and cause tumours which might be becomes cancer later on ${ }^{[8]}$. Several studies have shown that millimetre waves of low intensity causing a variety of biological changes, including cell membrane effects, also at non-thermal temperatures ${ }^{[3]}$. It appears that the $95 \mathrm{GHz}$ millimetre wave's frequency affects the cutaneous nociceptors and act as a threatening stimulus without heating or thermal damage ${ }^{[9]}$. Short term application of certain frequencies stimulates a release of endogenous opioids in the skin. The sweat canals, filled with a conductive aqueous solution in the skin can act as antennas and thus respond to millimetre waves ${ }^{[4]}$.

\section{Effects on eyes}

Cataract has remained a leading cause for blindness. Age, obesity, asthma, smoking, diabetes, and type B ultraviolet radiation are well known risk factors for the formation of cataracts. Radiation from the microwave is also a frequent cause of cataracts. The eyes may also receive significant radiation, especially in nearby field exposures. Microwave radiation is also a known cause of cataracts with heat being an undisputed mechanism. The eyes lack sufficient blood flow to dissipate heat effectively ${ }^{[3-4]}$.

\section{Neurological effects}

When the nervous system or brain is disrupted, morphological, electrophysiological and chemical alterations can occur that may be observed by Electronic Medical Records (EMR). A significant change in these functions will inevitably lead to a change in behaviour. Indeed, the neurological effects of EMR reported in the literature include changes in blood-brain-barrier, morphology, electrophysiology, neurotransmitter functions, cellular metabolism, calcium efflux, responses to drugs that affect the nervous system ${ }^{[10]}$.

\section{Effects of $5 \mathrm{G}$ radiations on plants}

Exposure to radio frequencies causes necrosis in leaves and low-intensity millimetre waves invoke peroxidase (a stress protein in plants) isoenzyme spectrum changes in ${ }^{[4]}$. The level of damage due to radiation in a plant is determined by the radiation absorbed by the plant and the time it is exposed to it. Following are the harmful impacts on plants species with elevated doses of radiation ${ }^{[3]}$ :

- Chromosomal aberrations identified in the chromosome structure as visually visible changes.

- DNA injury, identified as harm to DNA molecules, including "inversion" of the DNA sequence, and portions of "deleted" sequences.

- The decrease in growth described as a decrease in organisms' growth rate.

- Fertility symptoms such as sterility diminished reproductive capacity and developmental abnormality, and limited infant viability.

- Seed germination reduced.

- The direct explosion may cause tissue burn damage.

\section{Effect of 5 G radiation on biodiversity and ecosystem}

The population of birds and other species were decreased or disappeared from regions polluted with "non-ionizing" 4G radiations. Our machines destroy innocent living beings with their hideous manmade frequencies. No organism can tolerate $5 \mathrm{G}$ radiation in any quantity of time ${ }^{[3]}$.

Wildlife Biologist, Alfonso Balmori, said that Since 3G technology arrives, birds losing their nests and health issues recorded such as plumage loss, locomotion problems, reduced survivorship, and death have risen. The Birds influenced by millimetre wave's radiation include Rock Doves, House Sparrows, White Storks, Magpies and Collared Doves. A $5 \mathrm{G}$ trial in the Netherlands killed 287 birds in December, $2018^{[3]}$. Exposure to radiation from a mobile tower for just 5-30 minutes, the eggs of sparrows was disfigured ${ }^{[11]}$. The disfiguration of birds exposed for such a short amount of time to these frequencies is significant considering that the new $5 \mathrm{G}$ network will have a much higher density of base stations (small cells) throughout areas needing connection. The potential dangers of having so many small cells all over areas where birds live could cause whole populations of birds to have mutations that threaten their population's survival.

Not only birds the population of bee also diminished by the non-ionizing EMF radiation ${ }^{[6]}$. Warnke found that cellular devices had a detrimental impact on bees ${ }^{[12]}$. It reduces the egg-laying abilities of the queen leading to a decline in 
colony strength ${ }^{[4]}$. Colony collapse disorder is when many of the bees living in the hive abandon the hive leaving the queen, the eggs, and a few worker bees ${ }^{[11]}$. The worker bees exposed to this radiation also had worsened navigational skills, causing them to stop returning to their original hive after about ten days. Bees are an incredibly important part of the earth's ecosystem. Around one-third of the food produced today is dependent on bees for pollination, making bees are a vital part of the agricultural system ${ }^{[13]}$. Bees not only provide pollination for the plant-based food we eat, but they are also important to maintaining the food livestock eats. Without bees, a vast majority of the food eaten today would be lost or at the very least highly limited. Climate change has already caused a large decline in the world's bee population.

The impact that the cell towers have on birds and bees is important to understand, because all ecosystems of the earth are interconnected. If one component of an ecosystem is disrupted the whole system will be affected. The disturbances of birds with the cell towers of today would only increase, because with $5 \mathrm{G}$ a larger number of small cell radio-tower-like devices would be necessary to ensure high quality connection for users. Having a larger number of high concentrations of these millimetre waves in the form of small cells would cause a wider exposure to bees and birds, and possibly other species that are equally important to our environment.

\section{Thermal effects}

When electromagnetic radiation (EM) radiation is absorbed, it is converted into heat. As a result of a temperature change, biological processes alter their functions as a result of a change in temperature. If electromagnetically radiated particles oscillate and gain energy, this allows them to oscillate. The ultimate fate of this energy depends on the situation. It could be immediately reradiated and appear as scattered, reflected, or transmitted radiation. It may also get dissipated into other microscopic motions within the matter, coming to thermal equilibrium and manifesting itself as thermal energy in the material.

Intense radio waves can thermally destroy the living tissue. Including lasers, visible and ultraviolet lasers with ample energy can also quickly set fire to the paper. Ionizing electromagnetic radiation will create high-speed electrons in a sample/matter and break chemical bonds, but after these electrons collide many times with other atoms in the material eventually most of the energy gets converted to thermal energy, this whole process happening in a tiny fraction of a second ${ }^{[14]}$.

The thermal radiation is responsible for the inverse or timereversed absorption process. The resulting radiation may subsequently be absorbed by another piece of matter, with the deposited energy heating the material. Radiation is an important mechanism of heat transfer.

\section{Specific absorption rate (SAR)}

The rate at which radiation is absorbed by the human body is measured by specific absorption rate (SAR). It is expressed in units of watts per $\mathrm{kg}(\mathrm{W} / \mathrm{kg})$ of tissue. If the amount of heat generated is small, it can be dissipated by the body's thermoregulatory mechanism without causing adverse effects. If the temperature exceeds this capacity about 1 to 2 degrees Celsius, tissue damage may occur. Every mobile phone has a SAR ranking (although some manufacturers try to hide it). Regulating agencies has set its maximum levels for handsets. Radiations depend on its design, size, antenna, and how it is held and used.

Institute of Electrical and Electronics Engineers (IEEE) Committee on Man and Radiation, national and international organizations have established safety guidelines for exposure to RF energy. Testing has shown a wide range in radiation exposure to the user of various brands and models of mobile phones and tablets. Tests have demonstrated a wide variety of responses to radiation from the customers of various mobile phone manufacturers and models. No epidemiological studies on wireless communication for this spectrum range between 6 and $100 \mathrm{GHz}$ should be recalled [15].

SAR levels are used for mobile phones, tablets, and other handheld wireless devices to determine regulatory compliance. For millimetre wavelength devices and infrastructure power density above $6 \mathrm{GHz}$ (FCC) and above $10 \mathrm{GHz}$ (ICNIRP) needs to be measured with power density (FCC, 1997; Wu et al., 2015a) ${ }^{[16]}$ This is due to the higher energy absorption in a shallow area that causes heating more rapidly resulting in much higher SAR levels. The FCC maximum permissible exposure (MPE) in terms of power density for frequencies between 1.5 and $100 \mathrm{GHz}$ is 10 $\mathrm{mW} / \mathrm{cm}^{2}$ over a $30 \mathrm{~min}$ period ${ }^{[16]}$. Heat generated is a concern in handheld devices for $5 \mathrm{G}$ but is still considered the only valid measure of harm, no biological cellular alterations are considered ${ }^{[4]}$.

\section{Possible solutions}

Radio Frequency (RF) is widely known as a powerindependent technology for the Internet of Things (IoT) system ${ }^{[17]}$. The RF down converter is necessary to boost the wireless system's dynamic range, while the mixer stage is also a bonus for the entire Wi-Fi network to make a significant contribution to the total gain ${ }^{[18]}$. The Radiofrequency micro electro mechanical systems (RF MEMS) technology is emerging as a critical approach for meeting the challenging specifications of passive devices and networks of the next fifth (5G) generation technologies, including elevated operating frequencies, large tuning capability, decreased hardware reliability and low power consumption ${ }^{[19]}$. Aside from RF wireless techniques, which in particular were banned by international treaties as a tool of military-grade, we should establish alternate approaches based on proven electromagnet theories [20]. Possible approaches to implement modern methods $5 \mathrm{G}$ of connectivity that does not require wires or RFs.

The RNB consists of modulation of magnetic field lines free of curls (CFs) that stretch in line with infinity and reach all intermediates. $\mathrm{CF}$ communication is a directional controlling system. The advantage of CF interaction is that it propagates faster than light. The downside is that the device is not appropriate for general public use ${ }^{[3]}$.

The other method is quantum communications. This approach will provide for any individual with an endless number of limitless bandwidth channels. This approach is much quicker than light for contact. It does not require radio waves or cables. The optimal confidentiality of information provided by quantum communications helps each customer directly. Quantum communications in no way threaten the life or the climate. Quantum occurrences, however, were just a part of the natural world ${ }^{[3]}$. 


\section{Conclusion}

The new 5G network will undoubtedly benefit consumers greatly, looking at 5G's long-term environmental impacts is also very important so that the risks are clearly understood and articulated.

In the age of emerging communication technologies, it has more disadvantages than the advantages, and it has often become life-threatening as well. In 5G technology, millimetre wave provide high data levels and establishes a link with all IOTs that can be considered beneficial, but the negative effects of $5 \mathrm{G}$ MMW on human health and biodiversity are not negligible, if we try to decrease the effects by considering the value of SAR then 5G technology can be considered as fruitful.

Since $5 \mathrm{G}$ is a new technology, its long-term effects on the environment are unknown. However, there are already concerns that $5 \mathrm{G}$ could have negative effects on the environment because of its energy use, and the impacts of manufacturing new infrastructure and a multitude of new devices.

The study aims to prove that Wireless Technology is one of the most damaging threats to the environment and wellbeing and the undermining personal freedom ever created without any solution other than termination. In humans, $4 \mathrm{G}$ and $5 \mathrm{G}$ cause multiple diseases and can kill everything. However, the review suggests some possible solutions that make $5 \mathrm{G}$ with no damage and available to the public also.

\section{Acknowledgement}

Authors acknowledge the immense help received from the scholars whose articles are cited and included in references of this review paper. The authors are also grateful to authors/ editors/ publishers of all those articles, journals and books from where the literature foe this article has been reviewed and discussed.

\section{References}

1. Nordrum Amy, Clark Kristen, IEEE Spectrum Staff. Everything You Need to know about 5G: Millimeter waves, massive MIMO, full duplex, beamforming, and small cells are just a few of the technologies that could enable ultrafast 5G networks. IEEE Spectrum 2017.

2. Nordrum, Clark, IEEE Spectrum Staff 2017.

3. Zaki U, Zaki MA, Shah SRA. Harmful effects of $5 \mathrm{G}$ on life with possible solution. Global Scientific Journal 2020;8(5):1-13.

4. Verma SC, Tejaswini TM, Pradhan D. Harmful effects of $5 \mathrm{G}$ radiations. Proceedings of IRAJ International Conference, 24th March, 2019, Bengaluru, India 2019, 71-75.

5. Segan Sascha. What is 5G? PC Magazine 2019.

6. Kostoff RN. "Adverse Effects of Wireless Radiation Copyright RN Kostoff 2019, 1-648.

7. Gorlach A, Bertram K, Hudecova S, Krizanova O. Calcium and ROS: A mutual interplay. Redox Biol 2015;6:260-271. Doi: 10.1016/j.redox.2015.08.010

8. Christianto V, Rn B, Smarandache F. "Environment: A Preliminary Review BAOJ Cancer Research \& Therapy Wireless Technologies (4G, 5G) Are Very Harmful to Human Health and Environment: A Preliminary Review, " 2019;5:4-7.

9. Keogh B, Zhu A. "Wideband Self-Interference Cancellation for 5G Full-Duplex Radio Using a Near-
Field Sensor Array, ” 2018 IEEE MTT-S Int. Microw. Work. Ser. 5G Hardw. Syst. Technol. IMWS-5G 2018;1:1-3. doi: 10.1109/IMWS-5G.2018.8484398.

10. Kish A, Aerospace O. "Fifth Generation Wireless (5G) Effects on Human Biology" 2019, 0-39.

11. Sivani S, Sudarsanam D. Impacts of radio-frequency electromagnetic field (RF-EMF) from cell phone towers and wireless devices on biosystem and ecosystem- a review. Biology and Medicine 2013;4(6):207.

12. Warnke Ulrich. Birds, Bees and Humankind: Destroying Nature by 'Electrosmog'. Competence Initiative for the Protection of Humanity, Environment and Democracy 2009.

13. Packham Chris. Would We Starve Without Bees? BBC. http://www.bbc.co.uk/guides/zg4dwmn

14. Commission FC, Wheeler T. "The Dangers Of $5 \mathrm{G}-11$ Reasons To Be Concerned," 2016, 2016-2019.

15. Abbasi MAB, Tataria Fusco H, VF, Matthaiou M. "On the Impact of Spillover Losses in $28 \mathrm{GHz}$ Rotman Lens Arrays for 5G Applications, " 2018 IEEE MTT-S Int. Microw. Work. Ser. 5G Hardw. Syst. Technol. IMWS5G 2018, 1-3. doi: 10.1109/IMWS-5G.2018.8484443.

16. FCC Letter 5G Americas, 2016. FCC Filing 14-177 5G Americas. 2018. https://www.fcc.gov/ecfs/filin g/1093077796003/document/10930777 $96003462 \mathrm{f}$

17. Wagih M, Komolafe A, Zaghari, B, Weddell AS, Beeby S. "A broadband outlook on flexible and textile RF energy harvesting and wireless power transfer: from near-field to 5G A Broadband Outlook on Flexible and Textile RF Energy Harvesting and Wireless Power Transfer: from Near-Field to 5G" 2020, 5-6.

18. Bhaskar SCGM. "A3 stage RF down converter network for portable, wearable 5G applications" SN Appl. Sci 2020;2(1):1-17. doi: 10.1007/s42452-019-1850-0.

19. Persano A, Quaranta F, Taurino A, Siciliano PA, Iannacci J. "Thin Film Encapsulation for RF MEMS in 5G and Modern Telecommunication Systems" 2020.

20. Assimonis SD, Fusco V. "RF Energy Harvesting with Dense Rectenna-Arrays Using Electrically Small Rectennas Suitable for IoT 5G Embedded Sensor Nodes” 2018 IEEE MTT-S Int. Microw. Work. Ser. 5G Hardw. Syst. Technol. IMWS-5G 2018, 1-3. doi: 10.1109/IMWS-5G.2018.8484384. 